\title{
Hábitos alimentarios y su asociación con obesidad en escolares de Enseñanza Básica
}

\section{EATING HABITS AND OBESITY IN ELEMENTARY STUDENTS}

Aguilar $\mathrm{A}^{\mathrm{a}}$, Bustos $\mathrm{P}^{\mathrm{b}}$, Amigo $\mathrm{H}^{\mathrm{b}}$

Nutricionista.

Departamento de Nutrición, Facultad de Medicina, Universidad de Chile.

\section{Lic. Ana Carolina Aguilar Rabito}

Docente universitaria y Tutora de Trabajos de Investigaciones en la Carrera de Nutrición de la FCQ-UNA

\section{Descripción breve del Curriculum Vitae de la autora principal}

\section{Magíster Ana Aguilar Rabito}

Licenciada en Nutrición, egresada de la Universidad del Norte (2003).

Magíster en Ciencias Biológicas Mención Nutrición, egresada en la Facultad de Medicina de la Universidad de Chile (2007).

Especialista en Didáctica Universitaria, egresada en la Facultad de Ciencias Agrarias de la Universidad Nacional de Asunción (2007).

Coordinadora y Docente del Curso de Postgrado "Especialización en Dietética Clínica y Soporte Nutricional" en la Facultad de Ciencias Químicas de la Universidad Nacional de Asunción.

Miembro de la Comisión de Autoevaluación y de la Comisión Permanente de la Carrera de Nutrición de la Facultad de Ciencias Químicas de la UNA.

Miembro de la Sociedad Paraguaya de Nutrición.

Docente universitaria en la Carrera de Nutrición de la Universidad del Pacífico. 


\section{RESUMEN}

Introducción. Uno de los factores que determina obesidad es una alimentación hipercalórica y con desbalance en los macronutrientes los que a su vez están influidos por hábitos alimentarios inadecuados. Objetivo. Determinar los hábitos alimentarios de escolares de $2^{\circ}$ año básico y su asociación con el exceso de peso. Material y métodos. Se estudiaron 503 niños de segundo básico asistentes a escuelas municipales y colegios subvencionados, de siete comunas urbanas de Santiago (Chile); evaluados antropométricamente. Además, se recolectaron datos socioeconómicos y de alimentación (a través de una encuesta alimentaria de recordatorio de 24 horas y otra diseñada para conocer hábitos alimentarios). Los hábitos alimentarios y el consumo de algunos alimentos seleccionados fueron asociados a sobrepeso y obesidad mediante modelos de regresión múltiple. Resultados. La prevalencia del exceso de peso fue de $50,4 \%$. En conjunto, el consumo de verduras y frutas fue bajo, no superando los $240 \mathrm{~g}$ diarios, el de pan correspondió a $110 \mathrm{~g}$. Fue habitual el consumo de jugos y bebidas azucaradas que superaron los $400 \mathrm{ml}$ diarios. La cena constituyó un factor protector (OR: 0.553, IC: $0.339-0.902)$, mientras que un bajo consumo de lácteos durante el día (OR: 2.335, IC: $1.230-4.433$ ) y el consumo de lácteos enteros (OR: 1.665, IC: 1.025-2.704) en las colaciones constituyeron factores riesgo de obesidad. Estas asociaciones se dan principalmente en las niñas y en los escolares cuya actividad física es considerada más activa. Conclusión. Los hábitos alimentarios contribuyen en parte a explicar la obesidad de los escolares evaluados, aspecto necesario de considerar en intervenciones más integrales destinadas a prevenir y/o tratar este problema nutricional.

Palabras clave: hábitos alimentarios; escolares; obesidad.

\section{UNIBE $\mid \begin{aligned} & \text { REVISTA } \\ & \text { CIENTÍFICA }\end{aligned}$}




\section{ABSTRAC}

Introduction. One of the factors that determine obesity is a high caloric intake with a non balanced macronutrients contribution which depends on inadequate food habits. Objective. Determine the foods habits of school children from second level of elementary schools and its association with excess of weight. Material and methods. 503 children of second level, attending to public and subsidized schools from seven urban counties of Santiago (Chile) were anthropometrically evaluated. Socioeconomic and feeding information were collected (through a specially designed questionnaire and by a 24 hours recall). Feeding habits and the intake of some foods were associated to overweight and obesity through multiple regression models. Results. The prevalence of the excess of weight was $50.4 \%$. The intake of vegetables and fruits were low: $240 \mathrm{~g} /$ day (both) and the bread was $110 \mathrm{~g}$. Juices and sweetened drinks were frequently consumed been above $400 \mathrm{ml}$. Dinner constituted a protective factor of obesity (OR: 0.553 , CI: 1.339-0.902) while a low intake of dairy products (OR: 2.335, CI: 1.230-4.433) and the intake of whole milky foods as snacks (OR: 1.665, CI: 1.025-2.704) were risk factors of obesity. These associations are mainly in girls and in school children physically active. Conclusion. Food habits explain in part obesity in the school children of this study. This aspect should be considered in more integral interventions addressed to prevent or treat this nutritional problem.

Keywords: food habits, school, obesity. 


\section{INTRODUCCIÓN}

La niñez constituye una etapa crítica en el ser humano, que se caracteriza por crecimiento físico, desarrollo psicomotor y social, y por la adquisición de hábitos que condicionan la salud y bienestar en esta etapa de la vida ,así como también determinan la calidad de vida del adulto. ${ }^{1}$ Debido a ello, los niños forman un grupo vulnerable que requiere de atención especial e integral.

De las enfermedades nutricionales, se destaca la obesidad, que actualmente es un problema prevalente y de rápido aumento, tanto en la población adulta como infantil. ${ }^{2}$ En Chile, la prevalencia de sobrepeso y obesidad en menores de 6 años, es elevada y aumenta su frecuencia en edades posteriores, constituyendo un factor de riesgo importante de varias enfermedades crónicas no transmisibles del adulto. ${ }^{3}$

Uno de los retos nutricionales actuales, es evitar la aparición de la obesidad y/o disminuir las altas cifras observadas. Los factores que contribuyen a la aparición de esta patología en la niñez, son complejos y la interacción entre ellos juega un papel muy importante. Como parte de estos factores se pueden citar la herencia, el sedentarismo, el excesivo consumo de alimentos y hábitos alimentarios inadecuados. ${ }^{4}$

El comportamiento aprendido, juega un papel importante en la determinación del estado nutricional normal de la población y también de los problemas nutricionales debido a que, la adquisición de hábitos (actividad física, alimentación), se desarrollan en edades tempranas. ${ }^{56}{ }^{6}$ Teniendo en cuenta que el comportamiento aprendido condiciona las costumbres alimentarias, el propósito de este estudio fue evaluar si los hábitos alimentarios inadecuados contribuyen al desarrollo de la obesidad en niños que cursan segundo año básico.

\footnotetext{
' Rosso P. Aspectos biológicos del desarrollo. En: Meneghello J, Fanta E, Paris E, Rosselot J. Ed Mediterráneo, $4^{a}$ Ed. Santiago, 1991.

${ }^{2}$ Amigo H. Obesidad en el niño en América Latina: situación, criterios de diagnóstico y desafios. Cad Saúde Pública 2003; 19 (Sup 1): S163-S170.

${ }^{3}$ Albala C, Vio F, Kain J, Uauy R. Nutrition transition in Chile: determinants and consequences. Public Health Nutrition 2002; 5 (1A): 123-128.

${ }^{4}$ Reilly JJ. Obesity in childhood and adolescence: evidence based clinical and public health perspectives. Postgrad Med J 2006; 82:429-37.

${ }^{5}$ Nicklas T, Baranowski T, Cullen K, Berenson G. Eating patterns, dietary quality and obesity. J Am College Nutr 2001; 20 (6): 599-608.

${ }^{6}$ Nicklas T, Yang SJ, Baranowski T, Zakeri I, Berenson G. Eating patterns and obesity in children. Am J Prev Med 2003; 25 (1): 9-16.
}

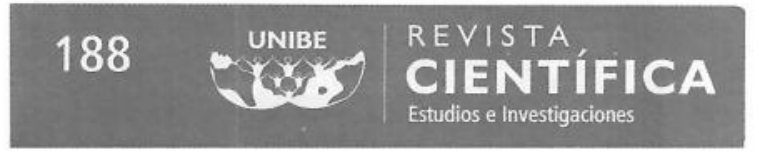




\section{MATERIALES Y MÉTODOS}

Estudio transversal, con datos primarios recolectados, durante el año 2006 en un estudio multinivel en escolares de Enseñanza Básica de escuelas básicas municipalizadas y particulares subvencionadas, que tuvieran alta, mediana y baja prevalencia de obesidad, de siete barrios de alta prevalencia la ciudad de Santiago de Chile: Independencia, Quinta Normal, Santiago Centro, Renca, Nuñoa, San Miguel y Lo Espejo.

Los sujetos de estudio fueron seleccionados mediante muestreo aleatorio simple, totalizando un total de 503 escolares de ambos sexos, independiente de su estado nutricional, pero teniendo en cuenta como mínimo un año de antigüedad en la escuela seleccionada, como criterio de inclusión. Como factor de exclusión se tuvo en cuenta que ningún escolar seleccionado, presentara alguna enfermedad que pudiera secundariamente determinar obesidad, o un mayor desarrollo puberal que lo esperado para la edad o alguna incapacidad física, que impidiera la realización de las mediciones antropométricas.

Para el tamaño muestral se consideró un $\alpha \leq 0,05$ y un poder del test del $80 \%$; teniendo en cuenta dos posibles asociaciones, alto número de colaciones ( $>3$ ) y saltarse algún tiempo de cena, basándose en resultados preliminares, que arrojaron una prevalencia de obesidad del $25 \%$.

Se tuvieron en cuenta datos antropométricos de peso y talla medidos según estándares internacionales; ; y también datos de alimentación, siendo utilizado para éste último un recordatorio de 24 horas y también una de hábitos diseñada para los fines del estudio (EAAN), el cual se complementó con datos básicos de actividad física y los demográficos de la familia y el escolar evaluado. Respecto a la alimentación, si el escolar evaluado era beneficiario del Programa de Alimentación Escolar (PAE), adicionalmente, se registraba si el consumo del plato servido era total, parcial o no lo consumió el día anterior a la encuesta.

El método de recolección de datos fue la entrevista personal a la madre, previa firma del consentimiento del informado, en presencia del escolar a ser evaluado.

De Onis M, Onyango AW, Van den Broeck J, Chumlea WC, Martorell R. Measurement and standardization protocols for anthropometry used in the construction of a new international growth reference. Food Nutr Bull 2004; 25 (1 Suppl): S27-36 
Se utilizó una balanza electrónica digital con antropómetro incorporado $\left(\right.$ Seca $\left.{ }^{\circledR}\right)$ calibrada periódicamente ${ }^{8}$, con capacidad máxima de 150 kilogramos y precisión de 100 gramos para el peso y para la talla dos metros de capacidad y precisión de de un $\mathrm{mm}$.

El estado nutricional se determinó utilizando el Índice de Masa Corporal (IMC) $\left(\mathrm{kg} / \mathrm{m}^{2}\right)$ que se comparó, según edad y sexo, con la referencia CDC/ NCHS 2000; disponible en el año de realización del estudio (2006). Los puntos de corte para clasificar el estado nutricional fueron los siguientes: bajo peso: IMC percentil < 10 , peso normal: IMC entre percentil 10 y 85 ; sobrepeso: IMC percentil entre 85 y 94 ; obesidad: IMC percentil $\geq 95$.

Los datos fueron digitalizados en ${ }^{\circledR}$ Epi Data $2.1 \mathrm{~b}$ y analizados en el programa estadístico ®SPSS para Windows. Version 14.0.1 (EE.UU.). Para el procesamiento de datos alimenticios se utilizó el software ${ }^{\circledR}$ SOFTNUT Versión 2005 (Progland Ltda., Chile).

Los datos cualitativos son expresados en frecuencia absoluta (n) y relativa $(\%)$, y los cuantitativos en promedio y desviación estándar para variables homogéneas y medianas con los valores límites para aquellas de distribución no homogénea. Para evaluar diferencias según tipo de variables, se utilizó la prueba $C h i^{2}\left(X^{2}\right)$, prueba de la mediana y ANOVA según corresponda. Se buscó asociaciones entre las variables independientes individualmente estudiadas con el estado nutricional; y finalmente un análisis en conjunto mediante una regresión logística múltiple, controlando por sexo, escolaridad materna, sedentarismo e ingesta calórica, condiciones con las que se exploró también su interacción con las variables independientes (hábitos alimentarios). Se consideraron significativos valores de $\mathrm{p} \leq 0,05$ para en todos los análisis.

Aspectos éticos de confidencialidad, respeto, beneficencia y justicia ${ }^{10}$ fueron considerados durante la investigación, y el estudio contó con la aprobación del Comité de Ética de la Facultad de Medicina de la Universidad de Chile.

${ }^{8}$ Habicht JP. Estandarización de métodos epidemiológicos cuantitativos sobre el terreno. Bol of Sanit Panam 1974; 76: 375-384.

${ }_{9}^{9}$ Ministerio de Salud 2004. Serie de Documentos Técnicos 1. Norma técnica de evaluación nutricional del niño de 6 a 18 años. Consultado en Octubre 2005. Disponible en: http://www. minsal.cl/ici/nutricion\%5CNormaEvNut6a18anos.pdf

${ }_{10}$ Adoptada por la $18^{\mathrm{a}}$ Asamblea Médica Mundial, Helsinki, Finlandia, Junio 1964 y enmendada por la $29^{\mathrm{a}}$ Asamblea Médica Mundial, Tokio, Japón, Octubre 1975, 35 $5^{\mathrm{a}}$ Asamblea Médica Mundial, Venecia, Italia, Octubre 1983, 41ª Asamblea Médica Mundial, Hong Kong, Septiembre $1989,48^{\mathrm{a}}$ Asamblea General, Somerset West, Sudáfrica, Octubre1996 y la $52^{\mathrm{a}}$ Asamblea General, Edimburgo, Escocia, Octubre 2000.

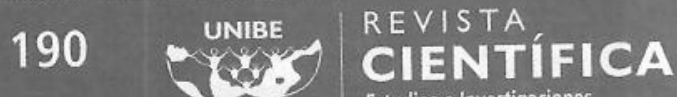




\section{RESULTADOS}

Este estudio se realizó con 503 escolares chilenos de Enseñanza Básica, de los cuales un porcentaje muy reducido presentó bajo peso $(3,2 \%)$, cerca de la mitad de ellos mostró un estado nutricional normal y la mitad restante exhibió exceso de peso, correspondiendo $25,2 \%$ a sobrepeso e igual porcentaje a obesidad; sin diferencias significativas por sexo.

Debido a que solo 16 niños, se hallaron con bajo peso y por la razón de que el estudio principalmente se focaliza en comparar los hábitos alimentarios en escolares que presentan exceso de peso y los que no lo presentan, los niños con bajo peso se agruparon en la categoría "sin exceso de peso" junto con los eutróficos agrupando al 49,5\% del total. En esta distribución, un poco más de la mitad corresponde a niñas $(53,4 \%)$, no habiendo diferencia significativa por sexo en ninguna categoría de estado nutricional (Gráfico 1).

\section{Gráfico 1. Estado nutricional de escolares por sexo}

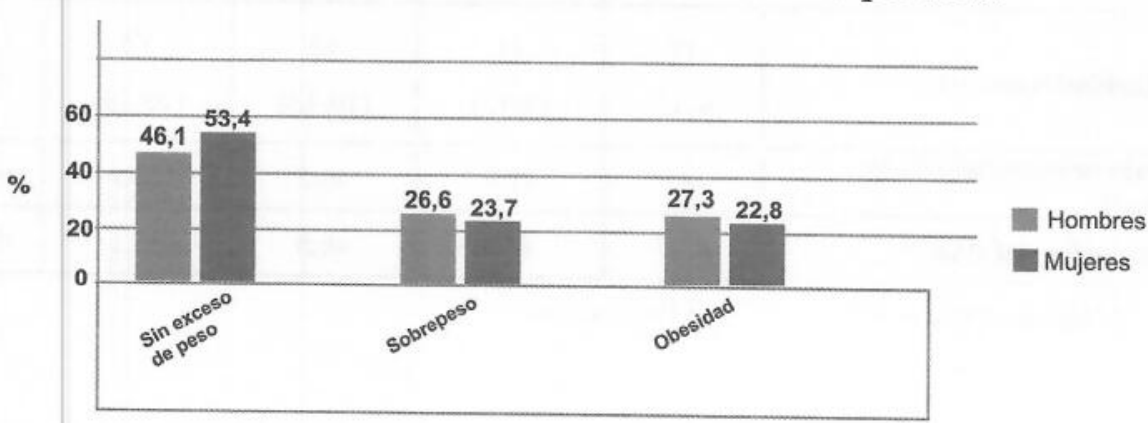

Entre las características generales se puede observar que los niños tienen una mediana de edad de 7,6 años (aspecto relativamente fijo por el diseño de la investigación); pertenecen a familias relativamente pequeñas, la escolaridad de la madre en promedio casi alcanza la educación media completa y la mitad de ellas realizaba trabajos remunerados fuera de la casa, aspectos que no difieren significativamente según estado nutricional.

El $43,7 \%$ de los escolares estudiados eran beneficiarios del PAE; llamando la atención que tienen mayor acceso a este programa los niños que no presentan exceso de peso $(47,8 \%)$ versus aquellos que presentan sobrepeso $(46,5 \%)$ y obesidad $(33,1 \%)(p=0,019)$ (Tabla 1$)$. 
Tabla 1. Descripción de la muestra por estado nutricional

\begin{tabular}{|c|c|c|c|c|c|}
\hline & $\begin{array}{c}\text { Total } \\
(\mathrm{n}=503)\end{array}$ & $\begin{array}{c}\text { Sin exceso } \\
\text { de peso } \\
(n=249)\end{array}$ & $\begin{array}{c}\text { Sobrepeso } \\
(n=127)\end{array}$ & $\begin{array}{c}\text { Obesidad } \\
(n=127)\end{array}$ & p valor \\
\hline Edad (años) ${ }^{s_{1}}$ & $\begin{array}{c}7,6 \\
(7,3-8,1)\end{array}$ & $\begin{array}{c}7,6 \\
(7,3-8,1)\end{array}$ & $\begin{array}{c}7,7 \\
(7,9-7,3)\end{array}$ & $\begin{array}{c}7.5 \\
(7,8-7,2)\end{array}$ & 0,259 \\
\hline Personas/hogar ${ }^{* 1}$ & $\begin{array}{c}5 \\
(4-6)\end{array}$ & $\begin{array}{c}5 \\
(4-6)\end{array}$ & $\begin{array}{c}5 \\
(4-6)\end{array}$ & $\begin{array}{c}5 \\
(4-5)\end{array}$ & 0,151 \\
\hline \multicolumn{6}{|l|}{ Tipo de familia ${ }^{32}$} \\
\hline Nuclear & 55,5 & 56,2 & 55,1 & 54,3 & \\
\hline Nuclear ampliada & 33,6 & 32,9 & 29,9 & 38,6 & 0,273 \\
\hline Extendida & 10,9 & 10,8 & 15,0 & 7,1 & \\
\hline Escolaridad materna ${ }^{* 1}$ & $\begin{array}{c}12 \\
(9-13)\end{array}$ & $\begin{array}{c}11 \\
(9-12)\end{array}$ & $\begin{array}{c}12 \\
(10-13)\end{array}$ & $\begin{array}{c}12 \\
(10-13)\end{array}$ & 0,096 \\
\hline $\begin{array}{l}\text { Trabajo remunerado de la } \\
\text { madre }^{*_{2}}\end{array}$ & 48,9 & 47,0 & 46,5 & 55,1 & 0,268 \\
\hline Beneficiarios del PAE ${ }^{* 2}$ & 43,7 & 47,8 & 46,5 & 33,1 & 0,019 \\
\hline
\end{tabular}

${ }^{* 1}$ Mediana (Espacio IC) $\quad{ }^{* 2}$ Porcentajes

En cuanto a la ingesta de calorías, se obtuvo que la mediana de ingesta de calorías diarias fue de 1721 , valores cercanos al promedio requerido para esta edad $\left(1700 \mathrm{Kcal} / \mathrm{d}^{11}\right)$. La ingesta de proteínas fue $59 \mathrm{~g}$, lo que determina alrededor de $2 \mathrm{~g} / \mathrm{kg}$ de peso y un P\% de 13,7. El consumo de hidratos de carbono representa el $60 \%$ y el de lípidos $27 \%$ de las calorías diarias (Tabla 2).

${ }^{11} \mathrm{FAO} / \mathrm{OMS} / \mathrm{UNU}$, Reporte final de Energía 2004. Ministerio de salud 2005. Guía de alimentación del niño(a), Guías de alimentación hasta la adolescencia 2005. Consultado en Abril 2006. Disponible en: http://www.minsal.cl/ici/nutricion/Guia_Alimentacion.pdf

\section{UNIBE, REVISTA


Tabla 2. Ingesta de energía y macronutrientes de escolares según R24 $\mathbf{H}$ por estado nutricional

\begin{tabular}{|c|c|c|c|c|c|}
\hline & Total & $\begin{array}{c}\text { Sin exceso } \\
\text { de peso }\end{array}$ & Sobrepeso & Obesidad & p valor \\
\hline Energía & $\begin{array}{c}1721,9 \\
(1462-2038)\end{array}$ & $\begin{array}{c}1735,1 \\
(1424-2083)\end{array}$ & $\begin{array}{c}1717,5 \\
(1436-2102)\end{array}$ & $\begin{array}{c}1705 \\
(1525-1966)\end{array}$ & 0,742 \\
\hline Proteínas & $\begin{array}{c}59 \\
(47,7-73)\end{array}$ & $\begin{array}{c}58,8 \\
(47,9-71,8)\end{array}$ & $\begin{array}{c}60 \\
(46,8-74,2)\end{array}$ & $\begin{array}{c}58,5 \\
(48,1-74,3)\end{array}$ & 0,754 \\
\hline Carbohidratos & $\begin{array}{c}258,6 \\
(213,6-305,6)\end{array}$ & $\begin{array}{c}259,3 \\
(210,5-311,8)\end{array}$ & $\begin{array}{c}255,5 \\
(206,9-300,6)\end{array}$ & $\begin{array}{c}261,5 \\
(226,3-302,5)\end{array}$ & 0,643 \\
\hline Lípidos & $\begin{array}{c}52,6 \\
(40,8-67,8)\end{array}$ & $\begin{array}{c}55,6 \\
(41,6-69,9)\end{array}$ & $\begin{array}{c}53,8 \\
(41,3-69,1)\end{array}$ & $\begin{array}{c}48,2 \\
(40,3-64,4)\end{array}$ & 0,062 \\
\hline
\end{tabular}

* Mediana (Espacio IC)

Según la opinión materna y según las horas que el niño pasa frente al televisor, en ambos casos son los niños obesos los más sedentarios $(p<0,001)$ (Gráfico 2).

\section{Gráfico 2. Sedentarismo}

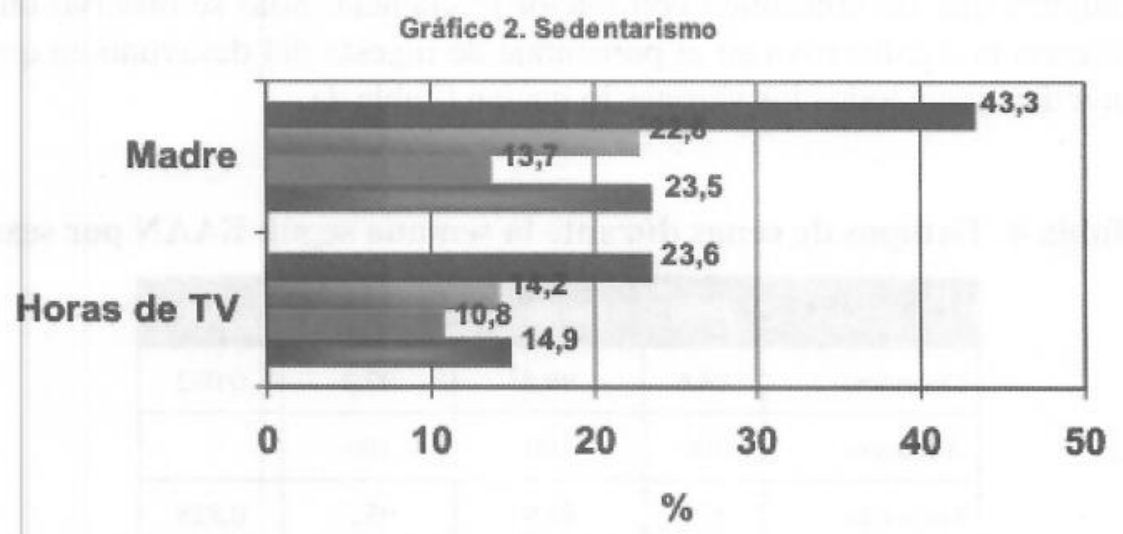

$p<0,001$

Total $\mathbf{\text { S/E peso }}=$ Sobrepeso Obeso 
Durante los días de semana, según la encuesta de los antecedentes alimentarios del niño, un porcentaje muy alto de los niños recibe desayuno, merienda y "colaciones" (sobre el 95\%), la totalidad de ellos almuerza, disminuyendo a la mitad la cifra de los que reciben cena. No se encontró diferencias significativas de estos valores según estado nutricional (Tabla 3 ).

Tabla 3. Tiempos de cenas durante la semana según EAAN por estado nutricional

\begin{tabular}{|l|c|c|c|c|c|}
\hline \multicolumn{2}{|c}{ Total $\begin{array}{c}\text { Sin exceso } \\
\text { de peso }\end{array}$} & Sobrepeso & Obesidad & p valor \\
\hline Desayuno & 98,6 & 98,8 & 99,2 & 97,6 & 0,529 \\
\hline Almuerzo & 100 & 100 & 100 & 100 & - \\
\hline Merienda & 95,6 & 95,2 & 96,9 & 95,3 & 0,737 \\
\hline Cena & 49,1 & 53,8 & 46,5 & 42,5 & 0,092 \\
\hline Colaciones & 95,6 & 94,8 & 96,1 & 96,9 & 0,625 \\
\hline
\end{tabular}

* Porcentajes

Se observó que un porcentaje levemente superior de hombres consume desayuno, cifra que se invierte en la cena y colaciones en que son las mujeres que las consumen con mayor frecuencia. Solo se observó una diferencia significativa en el porcentaje de ingesta del desayuno en que prácticamente todos los varones lo hacían (Tabla 4).

Tabla 4. Tiempos de cenas durante la semana según EAAN por sexo

\begin{tabular}{|l|c|c|c|c|}
\hline \multicolumn{2}{|c}{ Total } & Hombres & \multicolumn{1}{c|}{ Mujeres } & p valor \\
\hline Desayuno & 98,6 & 99,6 & 97,4 & 0,052 \\
\hline Almuerzo & 100 & 100 & 100 & - \\
\hline Merienda & 95,6 & 95,9 & 95,3 & 0,828 \\
\hline Cena & 49,1 & 47,2 & 51,3 & 0,372 \\
\hline Colaciones & 95,6 & 94,8 & 96,6 & 0,389 \\
\hline
\end{tabular}

* Porcentajes

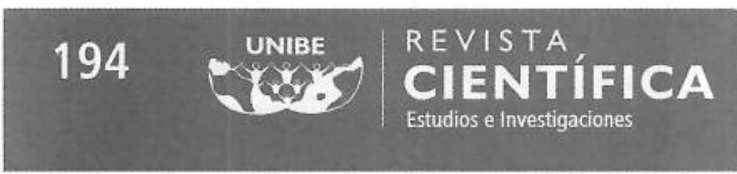


Durante el fin de semana, según la misma encuesta, existe una disminución leve en los porcentajes de los niños que realizan los diferentes tiempos de comidas. Los niños que no presentan exceso de peso son los que reciben mayormente la cena disminuyendo en aquellos con sobrepeso y obesidad $(p=0,038)$. También se redujo el porcentaje de los que consumen colaciones, aunque no se observaron diferencias significativas de este consumo según estado nutricional (Tabla 5).

Tabla 5. Tiempos de cenas durante el fin de semana según EAAN por estado nutricional

\begin{tabular}{lccccc}
\hline & Total & $\begin{array}{c}\text { Sin exceso } \\
\text { de peso }\end{array}$ & Sobrepeso & Obesidad & p valor \\
Desayuno & 93,4 & 95,6 & 92,1 & 90,6 & 0,139 \\
Almuerzo & 99,4 & 99,6 & 99,2 & 99,2 & 0,854 \\
Merienda & 93,6 & 93,6 & 92,9 & 94,5 & 0,875 \\
Cena & 41,0 & 46,6 & 36,2 & 34,6 & 0,038 \\
Colaciones & 74,6 & 77,1 & 73,2 & 70,9 & 0,390 \\
\hline
\end{tabular}

* Porcentajes

El análisis de los tiempos de comida en los fines de semana, según el sexo muestra la misma tendencia general de disminución, sin diferencias significativas entre hombres y mujeres (datos no presentados).

Durante la semana, según la ER24H se obtuvo que $94 \%$ de los niños desayunan, lo cual es levemente inferior a lo encontrado con la encuesta anterior, en que sólo 1,4\% no realizaba este tiempo de comida. Además, se pudo observar que 7,4\% efectúan dos desayunos, correspondientes a los niños que son beneficiados con el PAE. Prácticamente, todos reciben almuerzo y nuevamente, hay un grupo de niños que reciben doble almuerzo, que corresponden a los escolares beneficiados con el PAE (4,4\% del total). Con respecto a la merienda, se pudo observar que $10,7 \%$ no la recibe y $6,8 \%$ lo hace dos veces.

En la misma encuesta, se logró detectar que existen menos niños que cenan al compararlo con la encuesta anterior ( $35,2 \%$ vs. $41 \%$ ) observándose que si hay una tendencia significativa según estado nutricional, donde son los niños con sobrepeso y los obesos los que reciben menos frecuentemente este tiempo de cena $(p=0,018)$. 
Se constató igualmente que $2,8 \%$ de los niños no realiza merienda ni cena, el $70 \%$ hace una de ellas, y un $27,2 \%$ hace ambas comidas $(p=0,039)$. La distribución de estos tiempos de comida (merienda y cena) fue diferente según estado nutricional, observándose que los niños sin exceso de peso tienden a realizar ambos tiempos de cena y un porcentaje mayor de obesos tienden a saltarse estos tiempos. La distribución según esta encuesta se puede observar en la tabla 6 .

En este análisis se puede observar que los niños con exceso de peso, tienden a tener una peor distribución de los tiempos de comida en la tarde, mientras que aquellos con estado nutricional normal si bien tienden a tener mejor equilibrado estos tiempos de comidas, están realizando en mayor proporción la repetición de desayuno y almuerzo.

Tabla 6. Tiempos de cenas durante la semana según ER24H por estado nutricional

\begin{tabular}{|l|c|c|c|c|}
\hline \multicolumn{5}{|c}{ Total } \\
\multicolumn{2}{|c|}{ de peso } \\
\hline No desayuna & 6,2 & 4,8 & 6,3 & 8,7 \\
\hline 1 desayuno & 86,5 & 86,3 & 85,0 & 88,2 \\
\hline 2 desayunos & 7,4 & 8,8 & 8,7 & 3,1 \\
\hline p=0,186 & & & & \\
\hline No almuerza & 2,0 & 2,0 & 1,6 & 2,4 \\
\hline 1 almuerzo & 93,6 & 91,2 & 96,1 & 96,1 \\
\hline 2 almuerzos & 4,4 & 6,8 & 2,4 & 1,6 \\
\hline p=0,116 & & & & \\
\hline No toma merienda & 10,7 & 9,2 & 11,8 & 12,6 \\
\hline 1 merienda & 82,5 & 86,3 & 78,7 & 78,7 \\
\hline 2 merienda & 6,8 & 4,4 & 9,4 & 8,7 \\
\hline p=0,199 & & & & \\
\hline Cena & 35,2 & 40,2 & 32,3 & 28,3 \\
\hline p=0,018 & & & & \\
\hline No realiza merienda ni cena & 2,8 & 2,4 & 2,4 & 3,9 \\
\hline Merienda o cena & 70,0 & 64,3 & 74,8 & 76,4 \\
\hline Merienda y cena & 27,2 & 33,3 & 22,8 & 19,7 \\
\hline p=0,039 & & & & \\
\hline
\end{tabular}

\section{UniBE, $\begin{aligned} & \text { REVISTA } \\ & \text { CIENTÍFICA }\end{aligned}$


Los escolares acostumbran a tomar desayuno alrededor de las 8 horas, el almuerzo consumen a las 13 horas, realizan una merienda a las 19 horas y suelen realizar la cena en la noche a las 20:30 horas aproximadamente (Tabla 7).

Tabla 7. Horarios de las 4 cenas principales según ER24H por estado nutricional

\begin{tabular}{|l|c|c|c|c|}
\hline \multicolumn{2}{|c}{ Total } & $\begin{array}{c}\text { Sin exceso } \\
\text { de peso }\end{array}$ & Sobrepeso & Obesidad \\
\hline Desayuno & $\begin{array}{c}8: 00 \\
(7: 30-9: 30)\end{array}$ & $\begin{array}{c}8: 00 \\
(7: 20-9: 30)\end{array}$ & $\begin{array}{c}8: 00 \\
(7: 30-9 v 30)\end{array}$ & $\begin{array}{c}8: 00 \\
(7: 30-9: 30)\end{array}$ \\
\hline Almuerzo & $13: 00$ & $13: 00$ & $13: 00$ & $13: 00$ \\
$(13: 00-14: 00)$ & $(13: 00-14: 00)$ & $(13: 00-13: 00)$ & $(13: 00-14: 00)$ \\
\hline \multirow{2}{*}{ Merienda } & $19: 00$ & $18: 30$ & $19: 00$ & $19: 00$ \\
& $(18: 00-19: 30)$ & $(17: 30-19: 00)$ & $(18: 00-19: 30)$ & $(18: 00-19: 30)$ \\
\hline \multirow{2}{*}{ Cena } & $20: 30$ & $20: 00$ & $20: 00$ & $21: 00$ \\
& $(20: 00-21: 00)$ & $(20: 00-21: 00)$ & $(20: 00-21: 00)$ & $(20: 00-21: 00)$ \\
\hline
\end{tabular}

* Mediana (Espacio IC)

El horario de los que realizan otro desayuno es entre las 8 y 9 horas, el segundo almuerzo y merienda son un poco más tarde cercanos al horario del primero. El $46 \%$ de los niños toman desayuno fuera del horario definido como "ideal" (entre 7 y $8 \mathrm{~h}$ ), $5 \%$ realiza su almuerzo fuera de las 12 y $14,30 \mathrm{~h}$ lo mismo ocurre con la merienda donde el $58 \%$ no la realiza entre las 17 y las 18 horas y; en $12 \%$ la cena es en un horario diferente al arbitrariamente estipulado entre las 20 y las 21 horas (Gráfico 3). 
Gráfico 3. Comidas fuera de horarios según ER24H

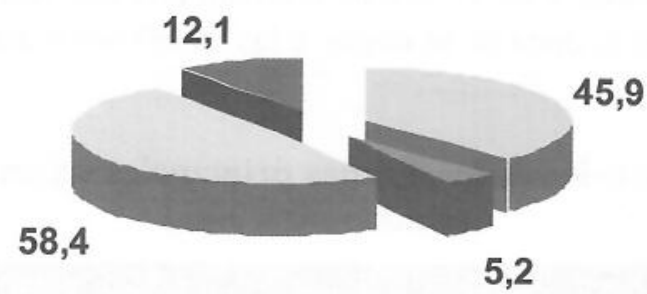

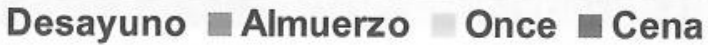

Las colaciones son más desordenadas. La más frecuente de las colaciones es la primera dándose en $62,6 \%$ de los niños independientemente del estado nutricional y sexo, realizándose la misma fijamente a las 10 horas de la mañana.

A partir de las 12 horas se observan varias colaciones llegando a ser según la ER24H como máximo seis, proveyéndose en distintos horarios (Tabla 8).

Tabla 8. Horarios de las colaciones según ER24h por estado nutricional

\begin{tabular}{|c|c|c|c|c|c|}
\hline & Total & $\begin{array}{c}\text { Sin exceso } \\
\text { de peso }\end{array}$ & Sobrepeso & Obesidad & $p$ valor \\
\hline $\begin{array}{l}\text { Colación I } \\
(n=315)\end{array}$ & $10,13 \pm 0,60$ & $10,10 \pm 0,52$ & $10,13 \pm 0,62$ & $10,20 \pm 0,73$ & 0,517 \\
\hline $\begin{array}{l}\text { Colación } 2 \\
(n=31)\end{array}$ & $11,96 \pm 1,10$ & $12,08 \pm 1,26$ & $11,87 \pm 0,88$ & $11,67 \pm 0,82$ & 0,723 \\
\hline $\begin{array}{l}\text { Colación } 3 \\
(\mathrm{n}=308)\end{array}$ & $15,86 \pm 0,94$ & $15,75 \pm 0,90$ & $15,99 \pm 10,3$ & $15,92 \pm 0,91$ & 0,152 \\
\hline $\begin{array}{l}\text { Colación } 4 \\
(\mathrm{n}=52)\end{array}$ & $17,13 \pm 1,40$ & $17,16 \pm 1,31$ & $16,63 \pm 1,66$ & $17,43 \pm 1,31$ & 0,305 \\
\hline $\begin{array}{l}\text { Colación } 5 \\
(\mathrm{n}=179)\end{array}$ & $20,55 \pm 1,30$ & $20,38 \pm 1,41$ & $20,58 \pm 1,09$ & $20,88 \pm 1,18$ & 0,111 \\
\hline $\begin{array}{l}\text { Colación } 6 \\
(\mathrm{n}=29)\end{array}$ & $20,80 \pm 4,02$ & $19,96 \pm 5,57$ & $21,61 \pm 1,44$ & $21,54 \pm 1,59$ & 0,575 \\
\hline $\begin{array}{l}\text { Colación } 7 \\
(\mathrm{n}=37)\end{array}$ & $21,88 \pm 10,6$ & $22,07 \pm 10,4$ & $21,36 \pm 1,16$ & $21,78 \pm 0,84$ & 0,259 \\
\hline
\end{tabular}

* Promedio \pm Desviación estándar

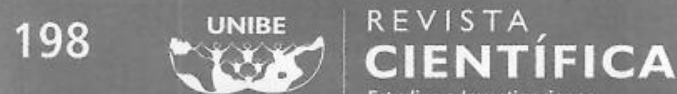


El consumo promedio de alimentos lácteos; los cuales incluyen leche, yogur, quesos; casi exclusivamente enteros; es de $470 \mathrm{ml}$ que es equivalente a dos tazas y un cuarto aproximadamente alcanzando el mínimo de lo recomendado para este grupo de edad. La ingesta diaria de las verduras y frutas es baja, no superando los 100 y $160 \mathrm{~g}$, respectivamente. En conjunto el consumo de verdura y frutas es de 238 $\mathrm{g}$ aproximadamente, siendo lo recomendado $\geq 400 \mathrm{~g} / \mathrm{d}$.

El consumo de pan corresponde a $110 \mathrm{~g}$, equivalente a una unidad. El consumo total de snacks alcanzó $85 \mathrm{~g}$, siendo relativamente mayor el consumo de snacks dulces con respecto a los salados. Es habitual el consumo de jugos y bebidas azucaradas superando los $400 \mathrm{ml} /$ día, representando en conjunto un aporte extra de 200 calorías/día.

No se encontró diferencia de consumo según estado nutricional, salvo en la ingesta de lácteos en que los niños sin exceso de peso tienden a consumir mayor cantidad que los obesos (Tabla 9).

Tabla 9. Consumo mediano diario de alimentos seleccionados según ER24H por estado nutricional

\begin{tabular}{|c|c|c|c|c|c|}
\hline Alimento & Total & $\begin{array}{c}\text { Sin exceso } \\
\text { de peso }\end{array}$ & Sobrepeso & Obesidad & p valor \\
\hline Lácteos (ml) & $\begin{array}{c}470 \\
(305-640)\end{array}$ & $\begin{array}{c}500 \\
(350-640)\end{array}$ & $\begin{array}{c}500 \\
(300-690)\end{array}$ & $\begin{array}{c}420 \\
(280-580)\end{array}$ & 0,020 \\
\hline Verduras (g) & $\begin{array}{c}78 \\
(62,5-132)\end{array}$ & $\begin{array}{c}75 \\
(70-120)\end{array}$ & $\begin{array}{c}80 \\
(60-141,4)\end{array}$ & $\begin{array}{c}80 \\
(50-145)\end{array}$ & 0,739 \\
\hline Frutas (g) & $\begin{array}{c}160 \\
(120-280)\end{array}$ & $\begin{array}{c}160 \\
(120-280)\end{array}$ & $\begin{array}{c}150 \\
(120-255)\end{array}$ & $\begin{array}{c}180 \\
(120-305)\end{array}$ & 0,158 \\
\hline $\operatorname{Pan}(\mathrm{g})$ & $\begin{array}{c}110 \\
(65-160)\end{array}$ & $\begin{array}{c}110 \\
(65-160)\end{array}$ & $\begin{array}{c}110 \\
(65-160)\end{array}$ & $\begin{array}{c}120 \\
(70-170)\end{array}$ & 0,946 \\
\hline Snack dulces (g) & $\begin{array}{c}45 \\
(36,8-92) \\
\end{array}$ & $\begin{array}{c}45 \\
(39-95) \\
\end{array}$ & $\begin{array}{c}45 \\
(40-100)\end{array}$ & $\begin{array}{c}40 \\
(20-75) \\
\end{array}$ & 0,181 \\
\hline Snack salados (g) & $\begin{array}{c}40 \\
(20-75) \\
\end{array}$ & $\begin{array}{c}38 \\
(12-82,5) \\
\end{array}$ & $\begin{array}{c}47,5 \\
(26,3-78,8)\end{array}$ & $\begin{array}{c}38 \\
(22-55) \\
\end{array}$ & 0,961 \\
\hline Jugos (ml) & $\begin{array}{c}200 \\
(180-400) \\
\end{array}$ & $\begin{array}{c}200 \\
(200-400) \\
\end{array}$ & $\begin{array}{c}300 \\
(180-450)\end{array}$ & $\begin{array}{c}200 \\
(180-400)\end{array}$ & 0,197 \\
\hline Bebidas (ml) & $\begin{array}{c}245 \\
(123,8-400)\end{array}$ & $\begin{array}{c}240 \\
(120-400)\end{array}$ & $\begin{array}{c}245 \\
(120-400)\end{array}$ & $\begin{array}{c}250 \\
(200-400)\end{array}$ & 0,964 \\
\hline
\end{tabular}

* Mediana (Espacio IC) 
Ahora bien teniendo en cuenta el sexo, los varones consumen significativamente mayor cantidad de pan, además que prefieren más los snack salados pero en este último caso no difiere estadísticamente con respecto a las mujeres (Tabla 10).

Tabla 10. Consumo de alimentos seleccionados según ER24h por sexo

\begin{tabular}{|c|c|c|c|c|}
\hline Alimento & Total & Hombres & Mujeres & p valor \\
\hline Lácteos (mL) & $\begin{array}{c}470 \\
(305-640)\end{array}$ & $\begin{array}{c}470 \\
(320-650)\end{array}$ & $\begin{array}{c}450 \\
(300-632,5)\end{array}$ & 0,951 \\
\hline Verduras (g) & $\begin{array}{c}78 \\
(62,5-132)\end{array}$ & $\begin{array}{c}80 \\
(65-140) \\
\end{array}$ & $\begin{array}{c}76 \\
(60-124) \\
\end{array}$ & 0,747 \\
\hline Frutas $(\mathrm{g})$ & $\begin{array}{c}160 \\
(120-280)\end{array}$ & $\begin{array}{c}150 \\
(120-277,5)\end{array}$ & $\begin{array}{c}160 \\
(120-280)\end{array}$ & 0,135 \\
\hline Pan (g) & $\begin{array}{c}110 \\
(65-160)\end{array}$ & $\begin{array}{c}127,5 \\
(81,3-180)\end{array}$ & $\begin{array}{c}100 \\
(60-150) \\
\end{array}$ & 0,0001 \\
\hline Snack dulces (g) & $\begin{array}{c}45 \\
(36,8-92) \\
\end{array}$ & $\begin{array}{c}47 \\
(36-100)\end{array}$ & $\begin{array}{c}45 \\
(37-80) \\
\end{array}$ & 0,896 \\
\hline Snack saldados (g) & $\begin{array}{c}40 \\
(20-75) \\
\end{array}$ & $\begin{array}{c}45 \\
(22-81,5) \\
\end{array}$ & $\begin{array}{c}35,5 \\
(12-52,5) \\
\end{array}$ & 0,371 \\
\hline Jugos (ml) & $\begin{array}{c}200 \\
(180-400)\end{array}$ & $\begin{array}{c}200 \\
(180-400)\end{array}$ & $\begin{array}{c}200 \\
(200-400)\end{array}$ & 0,081 \\
\hline Bebidas (ml) & $\begin{array}{c}245 \\
(123,8-400)\end{array}$ & $\begin{array}{c}240 \\
(120-400)\end{array}$ & $\begin{array}{c}250 \\
(162,5-400)\end{array}$ & 0,318 \\
\hline
\end{tabular}

* Mediana (Espacio IC)

Durante los días de semana y los fines de semana la mediana de colaciones fue 2 /día sin diferencias significativas según estado nutricional (Tabla 11) ni sexo (datos no presentados).

\section{UNIBE ${ }^{\text {REVISTA }}$ CIENTÍFICA


Tabla 11. Colaciones durante la semana según EAAN por estado nutricional

\begin{tabular}{|c|c|c|c|c|c|}
\hline & Total & $\begin{array}{c}\text { Sin exceso de } \\
\text { peso }\end{array}$ & Sobrepeso & Obesidad & $p$ valor \\
\hline $\begin{array}{l}\text { Colaciones } \\
\text { (Semana) }\end{array}$ & $\begin{array}{c}2 \\
(1-3)\end{array}$ & $\begin{array}{c}2 \\
(1-3)\end{array}$ & $\begin{array}{c}2 \\
(1-3)\end{array}$ & $\begin{array}{c}2 \\
(1-3)\end{array}$ & 0,916 \\
\hline $\begin{array}{l}\text { Colaciones } \\
\text { (Fin de } \\
\text { semana) }\end{array}$ & $\begin{array}{c}2 \\
(1-3)\end{array}$ & $\begin{array}{c}2 \\
(1-3)\end{array}$ & $\begin{array}{c}2 \\
(1-3)\end{array}$ & $\begin{array}{c}2 \\
(1-3)\end{array}$ & 0,494 \\
\hline
\end{tabular}

* Mediana (Espacio IC)

Sobre el 95\% de los niños ingiere más de una colación diaria. Solo un $4 \%$ consume una sola colación diaria, en donde es el $5,5 \%$ de los obesos los que mantienen esta rutina. El número de colaciones semanales comprendida entre los días de lunes a viernes llega a ser superior en 16 alimentos en el 25,2\% de los niños. Curiosamente los obesos no son los que consumen el más alto número de colaciones e incluso casi el doble de ellos recibe $<1$ colación/diaria. (Tabla 12).

Tabla 12. Número de colaciones según EAAN por estado nutricional

\begin{tabular}{|c|c|c|c|c|}
\hline $\begin{array}{c}\text { Colaciones } \\
\text { (n'/sem) }\end{array}$ & Total & $\begin{array}{c}\text { Sin exceso } \\
\text { de peso }\end{array}$ & Sobrepeso & Obesidad \\
\hline$\geq 16$ & 25,2 & 28,1 & 22,8 & 22,0 \\
\hline $11-15$ & 54,7 & 53,8 & 54,3 & 56,7 \\
\hline $6-10$ & 16,1 & 14,9 & 18,9 & 15,7 \\
\hline$\leq 5$ & 4,0 & 3,2 & 3,9 & 5,5 \\
\hline
\end{tabular}

* Porcentajes; * $\mathrm{p}=0,713$

Según antecedentes alimentarios del niño el 29,2\% de los niños come pan en sus colaciones y el consumo de dicho alimento es significativamente mayor en los obesos, sucediendo lo mismo en el caso de los alimentos con altas densidades energéticas provenientes de carbohidratos o grasas. La ingesta de fruta se da en un poco menos de la mitad de los escolares, y un porcentaje importante $(60,4 \%)$ realiza su colación con productos lácteos exclusivamente enteros (Tabla 13). 
Tabla 13. Tipo de colaciones semanales según EAAN por estado nutricional

\begin{tabular}{|c|c|c|c|c|c|}
\hline Alimento & Total & $\begin{array}{c}\text { Sin exceso } \\
\text { de peso }\end{array}$ & Sobrepeso & Obesidad & $p$ valor \\
\hline Pan & 29,2 & 27,7 & 22,0 & 39,4 & 0,008 \\
\hline Frutas & 45,9 & 46,6 & 46,5 & 44,1 & 0,862 \\
\hline Lácteos & 60,4 & 56,2 & 63,0 & 66,1 & 0,141 \\
\hline Snack & 16,5 & 18,9 & 9,4 & 18,9 & 0,047 \\
\hline
\end{tabular}

* Porcentajes

A través de la ER24H se analizó el tipo de alimento en la colación nocturna observándose que $7,2 \%$ de ellos consume frutas y $12,1 \%$ toma algún tipo de lácteos, ya sea, yogur o leche. De aquellos que consumen frutas $10,2 \%$ son obesos, disminuyendo su consumo en los con sobrepeso $(7,9 \%)$ y en los que no presentan exceso de peso $(5,2 \%)$. Los lácteos son más preferidos por los niños que no presentan exceso de peso (14,9\% vs. 8,7\%) (Gráfico 4).

\section{Gráfico 4. Tipo de colación nocturna según ER24H por estado nutricional}

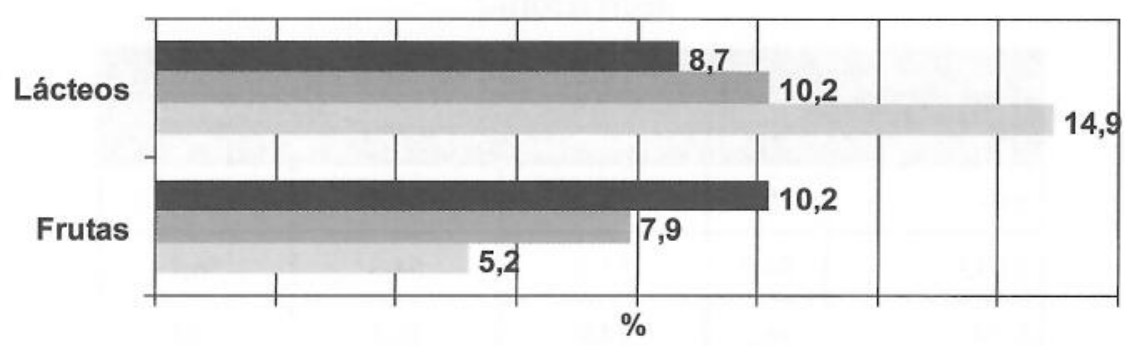

S/E Peso iㅣ Sobrepeso a Obeso

El 59\% de los niños realiza alguna colación saludable en el día. Es alta la prevalencia de niños que realizan colaciones no saludables durante el día (sobre $70 \%$ ) tanto en niños sin exceso de peso como los que presentan algún grado de exceso (datos no presentados).

Entre las variables utilizadas como control, que actúan sobre la variable dependiente pero no a través de las independientes, se pudo observar en los análisis univariados que-en esta muestra-el sexo y la ingesta de

\section{UNIBE ${ }^{\text {REVISTA }}$ CIENTÍFICA


calorías no tienen efecto en la determinación del exceso de peso, pero si lo tienen la mayor escolaridad materna y el sedentarismo. $\mathrm{Y}$ al buscar las asociaciones entre tiempos de cena y exceso de peso, se pudo observar que cenar en la noche aparece como un factor protector de obesidad $(\mathrm{p}=0,047)$ y el alto número de colaciones, como factor de riesgo de sobrepeso y obesidad ( $\mathrm{p}=0,049$ y 0,047 respectivamente).

En el modelo multivariado; ajustado por sexo, escolaridad materna, sedentarismo e ingesta de calorías; participan los mismos factores que se asociaron significativamente a obesidad en los análisis univariados (salvo el alto número de colaciones que se pierde) y en la misma dirección; es decir, resultando como factor protector consumir la cena y como factores de riesgo la baja ingesta de lácteos y la presencia de éste alimento en las colaciones (Tabla 14).

Tabla 14. Hábitos alimentarios y exceso de peso en escolares. Análisis multivariado

\begin{tabular}{|l|c|c|c|c|c|c|}
\hline \multicolumn{1}{|c}{ VARIABLES } & \multicolumn{1}{c}{ OR } & p valor & IC & OR & p valor & IC \\
\cline { 2 - 8 } & 0,686 & 0,114 & $0,430-1,095$ & 0,553 & 0,018 & $0,339-0,902$ \\
\hline Si cena & 2,080 & 0,022 & $1,112-3,889$ & 2,335 & 0,010 & $1,230-4,433$ \\
\hline Bajo consumo de lácteos $^{1}$ & 0,643 & 0,102 & $0,379-1,092$ & 1,590 & 0,063 & $0,975-2,594$ \\
\hline $\begin{array}{l}\text { Consumo de pan en } \\
\text { colaciones }\end{array}$ & 1,619 & 0,043 & $1,016-2,578$ & 1,665 & 0,039 & $1,025-2,704$ \\
\hline $\begin{array}{l}\text { Consumo de lácteos en } \\
\text { colaciones }\end{array}$ & & & & & &
\end{tabular}

* Ajustado por sexo, escolaridad materna, sedentarismo (h de TV) y calorías.

' Bajo consumo de lácteos $\leq 200 \mathrm{ml}$.

Se dejó en el modelo, el consumo de pan en las colaciones ya que en ésta muestra aparece como factor de riesgo especialmente en las mujeres; también se encontró que en hombres se pierden las asociaciones mientras, que en mujeres obesas los factores están más fuertemente asociados (Tabla 17). 
Tabla 17. Hábitos alimentarios y exceso de peso en escolares por sexo. Análisis multivariado

\begin{tabular}{|c|c|c|c|c|c|c|}
\hline \multirow{2}{*}{ VARIABLES } & \multicolumn{3}{|c|}{ SOBREPESO } & \multicolumn{3}{|c|}{ OBESOS } \\
\hline & OR & p valor & IC & OR & p valor & IC \\
\hline \multicolumn{7}{|l|}{$\begin{array}{l}\text { a) Modelo en } \\
\text { hombres }\end{array}$} \\
\hline Si cena & 0,771 & 0,429 & $0,404-1,470$ & 0,707 & 0,295 & $0,369-1,354$ \\
\hline $\begin{array}{l}\text { Bajo consumo de } \\
\text { lácteos }^{1}\end{array}$ & 2,280 & 0,063 & $0,958-5,429$ & 1,843 & 0,186 & $0,745-4,564$ \\
\hline $\begin{array}{l}\text { Consumo de pan } \\
\text { en colaciones }\end{array}$ & 0,360 & 0,009 & $0,167-0,777$ & 1,144 & 0,690 & $0,589-2,223$ \\
\hline $\begin{array}{l}\text { Consumo de } \\
\text { lácteos en } \\
\text { colaciones }\end{array}$ & 2,029 & 0,029 & $1,075-3,832$ & 1,794 & 0,072 & $0,948-3,394$ \\
\hline \multicolumn{7}{|l|}{$\begin{array}{l}\text { b) Modelo en } \\
\text { mujeres }\end{array}$} \\
\hline Si cena & 0,582 & 0,131 & $0,289-1,174$ & 0,408 & 0,021 & $0,190-0,875$ \\
\hline $\begin{array}{l}\text { Bajo consumo de } \\
\text { lácteos }^{1}\end{array}$ & 1,889 & 0,180 & $0,745-4,787$ & 2,897 & 0,024 & $1,147-7,318$ \\
\hline $\begin{array}{l}\text { Consumo de pan } \\
\text { en colaciones }\end{array}$ & 1,161 & 0,699 & $0,545-2,472$ & 2,435 & 0,020 & $1,149-5,162$ \\
\hline $\begin{array}{l}\text { Consumo de } \\
\text { lácteos en } \\
\text { colaciones }\end{array}$ & 1,291 & 0,474 & $0,642-2,600$ & 1,535 & 0,027 & $0,719-3,278$ \\
\hline
\end{tabular}

* Ajustado por escolaridad materna, sedentarismo (h de TV) y calorias.

' Bajo consumo de lácteos $\leq 200 \mathrm{ml}$.

La única interacción que hubo entre las variables de control y los hábitos alimentarios fue la observada entre sedentarismo y cena, por esta razón se realizó modelos diferenciados para los niños sedentarios y para los activos, observándose que en los primero se pierden las asociaciones, mientras que en los activos los hábitos alimentarios inadecuados aparecen como factores asociados significativamente tanto en sobrepeso como en obesos (Tabla 18).

\section{UNIBE $\mid$\begin{tabular}{l|l} 
REVISTA \\
CIENTÍFICA
\end{tabular}


Tabla 18. Hábitos alimentarios y exceso de peso en escolares según horas TV. Análisis multivariado

\begin{tabular}{|c|c|c|c|c|c|c|}
\hline \multirow{2}{*}{ VARIABLES } & \multicolumn{3}{|c|}{ SOBREPESO } & \multicolumn{3}{|c|}{ OBESOS } \\
\hline & OR & $\begin{array}{c}\text { p } \\
\text { valor }\end{array}$ & IC & OR & p valor & IC \\
\hline \multicolumn{7}{|l|}{$\begin{array}{l}\text { a) Modelo en } \\
\text { sedentarios }\end{array}$} \\
\hline Si cena & 5,154 & 0,039 & $\begin{array}{l}1,089 \\
24,401\end{array}$ & 1,654 & 0,418 & $0,490-5,586$ \\
\hline $\begin{array}{l}\text { Bajo consumo de } \\
\text { lácteos }^{1}\end{array}$ & 0,537 & 0,516 & $0,082-3,511$ & 3,003 & 0,151 & $0,670-13,464$ \\
\hline $\begin{array}{l}\text { Consumo de pan en } \\
\text { colaciones }\end{array}$ & 0,085 & 0,049 & $0,007-0,986$ & 1,511 & 0,546 & $0,395-5,785$ \\
\hline $\begin{array}{l}\text { Consumo de lácteos } \\
\text { en colaciones }\end{array}$ & 1,032 & 1,032 & $0,240-4,434$ & 1,299 & 0,663 & $0,401-4,207$ \\
\hline \multicolumn{7}{|l|}{$\begin{array}{l}\text { b) Modelo en } \\
\text { activos }\end{array}$} \\
\hline Si cena & 0,550 & 0,021 & $0,331-0,914$ & 0,445 & 0,004 & $0,255-0,775$ \\
\hline $\begin{array}{l}\text { Bajo consumo de } \\
\text { lácteos }^{1}\end{array}$ & 2,353 & 0,013 & $1,196-4,630$ & 2,104 & 0,047 & $1,010-4,382$ \\
\hline $\begin{array}{l}\text { Consumo de pan en } \\
\text { colaciones }\end{array}$ & 0,732 & 0,273 & $0,420-1,278$ & 1,673 & 0,061 & $0,976-2,868$ \\
\hline $\begin{array}{l}\text { Consumo de lácteos } \\
\text { en colaciones }\end{array}$ & 1,717 & 0,036 & $1,037-2,842$ & 1,703 & 0,054 & $0,991-2,927$ \\
\hline
\end{tabular}

* Ajustado por sexo, escolaridad materna y calorías.

${ }^{1}$ Bajo consumo de lácteos $\leq 200 \mathrm{ml}$. 


\section{DISCUSIÓN}

En este estudio se pudo constatar que los hábitos alimentarios son parcialmente responsables de la obesidad en niños de edad escolar, constituyendo la cena un factor protector frente al exceso de peso, mientras que un bajo consumo de lácteos, el consumo de pan y lácteos enteros en las colaciones constituyen factores riesgo de la obesidad. Estas asociaciones se dan principalmente en las niñas y en los escolares cuya actividad física es considerada más "activa", no así en los "sedentarios".

El que la cena sea un factor protector se puede interpretar a través de que el orden en las cenas juega un papel importante, al no ingerir a esa hora alimentos poco balanceados ya que la cena debiera contribuir a aportar entre otros, verduras; de esta manera estos alimentos no se recomendarían en un solo tiempo de cena (almuerzo) y estaría más acorde a la promoción actual que se está realizando para ellas ${ }^{12}$. Este tiempo de comida inclusive contribuye también a una mejor distribución de las calorías a lo largo del día.

El consumo de productos lácteos enteros y pan en las colaciones posiblemente tengan una explicación común; ambos cooperan aportando más calorías a partir de grasas y carbohidratos respectivamente, sobretodo en esta muestra en que se encontró que el consumo de lácteos, es casi exclusivamente a través de productos "enteros".

El que la asociación se observe en los escolares más activos plantea la posibilidad que en los sedentarios éste probablemente es el factor más determinante de exceso de peso (la inactividad) mientras que probablemente en los más activos, los hábitos alimentarios juegan un rol que contribuye en la mantención del exceso de peso. A su vez, esto es más relevante en las niñas porque justamente ellas son las más sedentarias.

A pesar de que no existe una definición aceptada de que es lo constituye "buenos hábitos alimentarios", al desglosar lo presentado por los escolares de esta muestra se podría comentar que:

- Tienen parcialmente adecuados tiempos de comida: sobre el $95 \%$ recibía desayuno, almuerzo y merienda. El ideal es que reciban también la cena, pero esto se observó en menos de la mitad de ellos, especialmente esto se dio en los obesos. Es importante recalcar que los niños tienen exceso de peso no solo por consumir ciertos

12 Zacarías I, Pizarro T, Rodríguez L, González D, Domper A. programa «5 AL DÍA» para promover el consumo de verduras y frutas en Chile. Rev. Chil. Nutr. 2006; 33 (Suppl 1): 276-280.

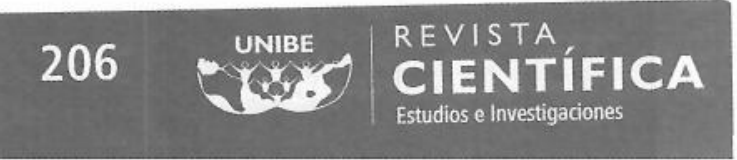


alimentos sino por la cantidad y la calidad de alimentos que reciben en cada tiempo de comida; por lo que resulta recomendable insistir que al tener un aporte de verduras las cenas son más reducidas en calorías pero principalmente en grasas. Realizando una buena combinación de alimentos se podría llegar al efecto de la saciedad para que no sea necesaria la presencia de una colación nocturna, ya que este tiempo de cena no sólo lo hacen en base a lácteos o frutas, sino muchos lo realizan con alimentos de altas densidades energéticas cuyas calorías extras no son necesarias. Esto destaca otro aspecto también necesario de considerar en la planificación de las comidas de los niños obesos que es el poder de saciedad de los alimentos que componen las mismas (aspecto no estudiado en este estudio) en que se ha descrito que los carbohidratos de digestión rápida tienen un poder de saciedad menor, especialmente en los obesos, que indica su menor regulación energética. ${ }^{13}$

Los resultados descriptivos demostraron que los escolares reciben dos veces al día algún tiempo de comida (niños que forman parte del Programa de Alimentación Escolar), lo cual no es conveniente. Esto lleva a una distorsión del objetivo del Programa y también a un aporte excesivo de alimentos y, por lo tanto, de calorías.

Los horarios de los principales tiempos de comidas son adecuados a pesar de la gran diversidad encontrada que obedece principalmente al tipo de jornada que tienen en la escuela. A futuro, cuando la jornada completa sea obligatoria para todos, esto puede también regularizarse más.

Definitivamente es inadecuado el alto número de colaciones que los niños llevan a la escuela al igual que el tipo de ellas. El que en cada recreo consuman uno o dos productos pareciera obedecer a la presión de los pares, al valor que le da la madre a que el niño lleve colación y también probablemente a la propaganda de estos productos, más que a la sensación de hambre, especialmente en aquellos que reciben doble desayuno ya que no pasan muchas horas entre una cena y otra. Resulta interesante observar el alto consumo de lácteos en las colaciones, especialmente yogurt, el que podría aún ser mejor si se consumiera bajo en materia grasa y sin adición de cereales, ya que éstos precisamente no son los adecuados al tener azúcar ó chocolate

$1 \$$ Alviña M, Araya H. Rapid carbohydrate digestion rate produced lesser short-term satiety in obese preschool children. Eur J Clin Nutr 2004; 58:637-642. 
adicional, por ejemplo. La ingesta de frutas, probablemente refleja más bien la colación que traen de la casa a una edad en que aún no manejan mucho dinero para hacer su propia elección de compra en los kioscos de la escuela. Se observó también que los niños obesos son los que mayormente consumen pan de colación lo cual es marcadamente un factor de riesgo en los análisis realizados en las niñas. Un estudio realizado en adultos muestra que el consumo de pan refinado (que es el que consumen los escolares evaluados) se asocia a ganancia de la circunferencia de cintura. ${ }^{14}$ En este estudio destaca lo inadecuado del consumo de colaciones con alto aporte calórico como es el caso de una enorme variedad de caramelos, chocolates y productos que combinan un bizcocho con agregado de manjar, chocolate o ambos. Estos productos, altamente preferidos por los niños ${ }^{15}$, se caracterizan por el aporte de carbohidratos simples, especialmente azúcar y también grasas, contribuyendo en la proporción total de lípidos consumidos en el día como se ha reportado en otros estudios ${ }^{16}$.

El que disminuya el número de colaciones el fin de semana puede ser un comportamiento habitual pero, igualmente puede ser que durante la semana la madre sea quien agregue la colación del niño y tenga "control" de lo que el niño lleva, mientras que cuando el niño está en la casa, puede comer diversos alimentos entre las cenas principales sin que ella repare en ello y que esto también esté subestimado al ser frecuente que los fines de semana los niños visiten sus familiares o salgan fuera de la casa consumiendo alimentos pero no quedando registrados en la memoria de la madre.

- Por otra parte, el consumo de bebidas gaseosas y jugos artificiales no resultó estar asociado a obesidad, pero es importante recalcar que existe un consumo elevado de ellos; coincidiendo con lo reportado en

\footnotetext{
${ }^{14}$ Halkiaer J, Sorensen TI, Tjonneland A, et al. Food and drinking patterns as predictors of 6-year BMI-adjusted changes in waist circumference. Br J Nutr 2004; 92: 735-748.

${ }^{15}$ Olivares S, Yáñez R, Díaz N. Publicidad de alimentos y conductas alimentarias en escolares de $5^{\circ}$ a $8^{\circ}$ básico. Rev Chil Nutr 2003; 30:36-42.

${ }^{16}$ Farris RP, Cresanta JL, Frank GC et al. Dietary studies of children from biracial population: intakes of fat and fatty acids in 10 and 13 year old children. Am J Clin Nutr 1984; 39:114-128.
}

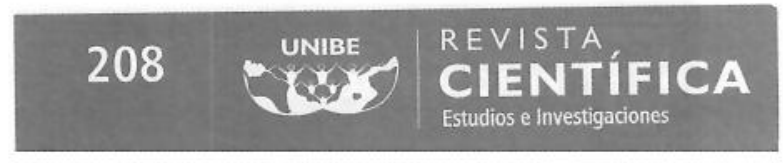


estudios previos ${ }^{17.18,19}$; estos aportan como mínimo 200 calorías extras al conjunto global de alimentación, calorías innecesarias además de poco recomendables por su alto índice glicémico ${ }^{20}$ más aún que si se los utiliza como sustitutos de alimentos como la leche o jugos de fruta natural o la fruta propiamente tal, ya que las bebidas no solo son utilizadas en las colaciones sino suele ser un acompañamiento en las cenas.

Con respecto a la ingesta de lácteos, destaca que su consumo es un factor protector de obesidad. Sin embargo, al igual que lo reportado en otros estudios nacionales e internacionales ${ }^{21,22,23}$ su ingesta es inferior a lo recomendado para los niños de este grupo de edad lo que plantea la permanente necesidad de estimular su consumo.

El consumo de pan fue menor a lo encontrado en investigaciones afines $^{24,25}$. Este alimento es básico en la cultura occidental, ya sea en el desayuno, como acompañamiento de las comidas principales, en sándwich, y es aquí donde aparentemente su consumo está asociado a obesidad especialmente en las niñas.

Se analizó la asociación de hábitos alimentarios con exceso de peso, encontrándose pobres resultados, pero es necesario recordar que los niños llegaron a su categoría de obesos o sobrepeso con bastante anterioridad a las encuestas realizadas para la investigación. Si hubo un cambio de hábitos al momento de pasar de normales a sobrepeso y a obesos se desconoce. Lo que se obtuvo es la existencia de hábitos alimentarios que permiten mantener la condición de exceso de peso.

17. Olivares S, Kain J, Lera L, Pizarro F, Vio F, Morón C. Nutritional status, food consumption and physical activity among Chilean school children: a descriptive study. Eur J Clin Nutr 2004; 58: 1278-1285.

${ }^{18}$ Olivares S, Zacarías I, Lera L, Leyton B, Durán R, Vío F. Estado nutricional y consumo de a limentos seleccionados en escolares de la Región Metropolitana: línea base para un proyecto de promoción del consumo de pescado. Rev Chil Nutr 2005; 32: 102-108.65.

${ }^{19}$ Olivares S, Bustos N, Moreno X, Lera L, Cortez S. Actitudes y prácticas sobre alimentación y actividad física en niños obesos y sus madres en Santiago, Chile. Rev Chil Nutr 2006; 33:
170-179.

${ }^{20}$ Sigman-Grant M, Morita J. Defining and interpreting intakes of sugars. Am J Clin Nutr 2003; 78: 815S-826S.

${ }^{21}$ Olivares S., et al., 2004.

22 Olivares S., et al., 2005.

${ }^{23}$ Olivares S., et al., 2006.

${ }^{24}$ Olivares S., et al., 2006.

${ }^{25}$ Atalah E, Urteaga C, Rebolledo A, Delfín S, Ramos R. Patrones alimentarios y de actividad física en escolares de la región de Aysén. Rev Chil Pediatr 1998; 70: 483-490. 
Como la focalización del PAE se basa en criterios sociales a fin de contribuir con la reducción de los niveles de ausencia y deserción escolar, permite incluir niños con obesidad; no obstante, en ellos sería muy importante poder entregar una alimentación más adaptada a sus necesidades ofreciendo opciones de almuerzo o desayuno a base de alimentos hipocalóricos, como ser por ejemplo, en vez de entregar leche entera con pan y mermelada, podría sustituirse por lácteos bajos en grasas y una fruta ya en los escolares mayores y sobre todo si de este programa se benefician también adolescentes. También habría que insistir en la no existencia de duplicación de los tiempos de comida, es decir, en la casa y luego en el ámbito escolar o viceversa.

En lo referente a colaciones, es importante que sea enviada de la casa evitando entregar dinero para no influir en la compra de alimentos no saludables, ya que la mayoría de las escuelas cuenta con cantinas o kioscos cuya venta de alimentos muchas veces no son las más adecuadas o a un costo accesible. Así mismo sería conveniente entregar un calendario de colaciones (como se realiza en la Educación Preescolar) que limita de alguna manera que las colaciones sean múltiples y favorece el consumo de frutas y lácteos.

A los autores del estudio les pareció que el tipo de análisis realizado, regresión politómica, permite comparar dos grupos de riesgo, en relación a los "de peso adecuado", ya que al agrupar a los niños que presentaban sobrepeso en una sola categoría, con los obesos la asociación habría perdido fuerza. No se observó una gradiente de riesgo como se hubiera querido hallar, que puede tener que ver con lo arbitrario, que son los límites que se consideran para agrupar en cada categoría pero también, porque los escolares con sobrepeso tienen conductas alimentarias más cercanas a las de los sin exceso de peso, que tampoco en la actualidad son tan saludables.

En el presente estudio, se pudo percibir algunas limitaciones propias de la metodología utilizada en la obtención de información así como en sus aplicaciones ${ }^{26,27}$.

Otra limitación a considerar es que las encuestas alimentarias realizadas para el estudio original no tenían como fin principal el estudio de los

${ }^{26}$ Frank GC, Berenson GS, Schilling PE, et al. Adapting the 24 hour recall for epidemiologic studies of school children. Jam Diet Assoc 1977; 71: 23-30.

${ }_{27}$ Dwyer JT, Kroll EA. The problem of memory in nutricional epidemiology research. Jam

Diet Assoc 1987; 87: 1509-1512.

\section{UNIBE $\mid$ REVISTA}


hábitos alimentarios; pero es conocido que el tipo de encuesta a aplicar debe estar en función de cuáles son los objetivos principales del estudio ${ }^{28}$. Además, las encuestas de recordatorios de 24 horas para estar debidamente representados debieran realizarse por lo menos dos días de la semana y uno de fin de semana, ya que la alimentación forma parte de las costumbres alimentarias y sociales, aparte de que existen cambios destacados en la dieta según el día de la semana. Por ejemplo, es habitual que los domingos y festivos se realicen ingestas más abundantes, que incluyan más productos dulces y de pastelería, que un día de semana, sobre todo en niños o adolescentes. También, se recomienda realizar la encuesta en dos periodos distintos del año, a fin de evitar diferencias por la estacionalidad ya que es conocida la variabilidad en el consumo energético y de nutrientes entre la época cálida y fría del año. Sin embargo, es necesario señalar que las encuestas de este estudio se realizaron en su totalidad entre mayo y septiembre del año 2005-2006 por lo que probablemente el efecto de la estacionalidad fue mínimo.

El realizar más de un tipo de encuesta para conocer los hábitos alimentarios es adecuado, ya que complementan la información aportada por la madre. Al preguntar por la alimentación del día anterior (ER24H), se pudo observar que la mayoría de las madres refieren la alimentación que el niño realiza en la casa, no reportando por ejemplo el segundo desayuno que el niño recibe a través del PAE, pero sí señalan el almuerzo del PAE, probablemente por considerarlo un tiempo de comida central e importante.

Entre los problemas del encuestado, aparece en este grupo un porcentaje de madres que trabajan $(48,9 \%)$, que muchas veces desconocen el detalle ya no alimentación de sus hijos, especialmente en horas en que el niño persona que tiene probablemente meno cuidado de algún familiar u otra y que provoca una subestimación de la inulación de las cenas del niño y un desconocimiento del aumen calorías y nutrientes si toman dos veces merienda, o siempos de cena del niño (e.g. pan u otros alimentos entre los no cuente exactamente tode caso en el estudio pudo ocurrir que el niño si algún compañero u todo lo que comió en la escuela, especialmente no sucede todos a convida alimentos, lo cual quizá estado nutricional en fías. De igual forma, podría haber un efecto del las madres de los niños reporte de alimentos consumidos; es decir que

${ }^{28}$ Rebolledo A. Encuesta alimentarias. Rev Chil Nutr 1998; 25: 28-34. 
comen, porque se espera que ellas tengan controlada la alimentación de sus hijos para conseguir un control del peso.

Hay que enfatizar que la recolección de datos y la interpretación de éstos por parte de los encuestadores también pueden implicar un riesgo de sesgos debido al efecto de la subjetividad tanto del encuestado como del encuestador ${ }^{29}$. Por lo anterior, es importante destacar la importancia de que la encuesta sea realizada principalmente a la persona que forma parte del estudio; sin embargo, se optó por encuestar a las madres y también a los escolares, porque los niños de 7 a 8 años todavía no pueden precisar cantidades o probablemente recordar la alimentación completa del día anterior.

\section{CONCLUSIONES}

Los hábitos alimentarios tienen aspectos positivos (adecuado número de comidas diarias y horarios de los tiempos de comidas) pero también algunos desfavorables como el consumo frecuente de colaciones durante el día, el bajo consumo de productos lácteos, verduras, frutas; y alta ingesta de jugos y bebidas azucaradas. La inactividad física es un factor de riesgo de obesidad.

Los hábitos alimentarios se asociaron parcialmente a obesidad al haberse encontrado una asociación inversa con el consumir cena y el bajo consumo de productos lácteos; y directa con el consumo de pan y lácteos enteros en las colaciones.

Estas asociaciones se dieron en escolares de sexo femenino y en los considerados más activos, antecedentes que permiten entregar elementos para intervenciones dirigidas a prevenir el exceso de peso en escolares desde que ingresan a la escuela.

${ }^{29}$ Urteaga C, Pinheiro AC. Investigación alimentaria: consideraciones practicas para mejorar la confiabilidad de los datos. Rev Chil Nutr 2003; 30:235-242.

\section{UNIBE REVISTA CIENTÍFICA}




\section{BIBLIOGRAFÍA}

Albala C, Vio F, Kain J, Uauy R. Nutrition transition in Chile: determinants and consequences. Public Health Nutrition 2002; 5 (1A): 123-128.

Alviña M, Araya H. Rapid carbohydrate digestion rate produced lesser short-term satiety in obese preschool children. Eur J Clin Nutr 2004; 58:637-642.

Amigo H. Obesidad en el niño en América Latina: situación, criterios de diagnóstico y desafíos. Cad Saúde Pública 2003; 19 (Sup 1): S163-S170.

Atalah E, Urteaga C, Rebolledo A, Delfín S, Ramos R. Patrones alimentarios y de actividad física en escolares de la región de Aysén. Rev Chil Pediatr 1998; 70: 483-490.

De Onis M, Onyango AW, Van den Broeck J, Chumlea WC, Martorell R. Measurement and standardization protocols for anthropometry used in the construction of a new international growth reference. Food Nutr Bull 2004; 25 (1 Suppl): S27-36.

Dwyer JT, Kroll EA. The problem of memory in nutricional epidemiology research. Jam Diet Assoc 1987; 87: 1509-1512.

FAO/OMS/UNU, Reporte final de Energía 2004. Ministerio de salud 2005. Guía de alimentación del niño(a), Guías de alimentación hasta la adolescencia 2005. Consultado en Abril 2006. Disponible en: http:// www.minsal.cl/ici/nutricion/Guia_Alimentacion.pdf

Farris RP, Cresanta JL, Frank GC et al. Dietary studies of children from biracial population: intakes of fat and fatty acids in 10 and 13 year old children. Am J Clin Nutr 1984; 39:114-128.

Frank GC, Berenson GS, Schilling PE, et al. Adapting the 24 hour recall for epidemiologic studies of school children. Jam Diet Assoc 1977; 71 : 23-30.

Habicht JP. Estandarización de métodos epidemiológicos cuantitativos sobre el terreno. Bol of Sanit Panam 1974; 76: 375-384.

Halkiaer J, Sorensen TI, Tjonneland A, et al. Food and drinking patterns as predictors of 6-year BMI-adjusted changes in waist circumference. Br J Nutr 2004; 92: 735-748.

Nicklas T, Baranowski T, Cullen K, Berenson G. Eating patterns, dietary quality and obesity. J Am College Nutr 2001; 20 (6): 599-608.

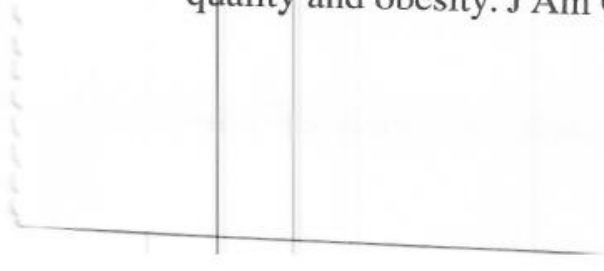


Nicklas T, Yang SJ, Baranowski T, Zakeri I, Berenson G. Eating patterns and obesity in children. Am J Prev Med 2003; 25 (1): 9-16.

Olivares S, Bustos N, Moreno X, Lera L, Cortez S. Actitudes y prácticas sobre alimentación y actividad física en niños obesos y sus madres en Santiago, Chile. Rev Chil Nutr 2006; 33: 170-179.

Olivares S, Kain J, Lera L, Pizarro F, Vio F, Morón C. Nutritional status, food consumption and physical activity among Chilean school children: a descriptive study. Eur J Clin Nutr 2004; 58: 1278-1285.

Olivares S, Yáñez R, Díaz N. Publicidad de alimentos y conductas alimentarias en escolares de $5^{\circ}$ a $8^{\circ}$ básico. Rev Chil Nutr 2003; 30:36-42. Olivares S, Zacarías I, Lera L, Leyton B, Durán R, Vío F. Estado nutricional y consumo de alimentos seleccionados en escolares de la Región Metropolitana: línea base para un proyecto de promoción del consumo de pescado. Rev Chil Nutr 2005; 32: 102-108.65.

Rebolledo A. Encuesta alimentarias. Rev Chil Nutr 1998; 25: 28-34.

Reilly JJ. Obesity in childhood and adolescence: evidence based clinical and public health perspectives. Postgrad Med J 2006; 82:429-37.

Rosso P. Aspectos biológicos del desarrollo. En: Meneghello J, Fanta E, Paris E, Rosselot J. Ed Mediterráneo, 4 ${ }^{a}$ Ed. Santiago, 1991.

Sigman-Grant M, Morita J. Defining and interpreting intakes of sugars. Am J Clin Nutr 2003; 78: 815S-826S.

Urteaga C, Pinheiro AC. Investigación alimentaria: consideraciones practicas para mejorar la confiabilidad de los datos. Rev Chil Nutr 2003; 30:235-242.

Zacarías I, Pizarro T, Rodríguez L, González D, Domper A. programa «5 AL DÍA» para promover el consumo de verduras y frutas en Chile. Rev. Chil. Nutr. 2006; 33 (Suppl 1): 276-280.

\section{UNIBE $\begin{aligned} & \text { REVISTA } \\ & \text { CIENTÍFICA }\end{aligned}$}

